\title{
The use of RF communication devices in ranging applications
}

\author{
Michał Śmieja \\ University of Warmia and Mazury in Olsztyn, Faculty of Technical Sciences, ul. Stoneczna 46A, 10-710 Olsztyn, Poland
}

\begin{abstract}
Positioning systems based on observation of satellites are still efectively developed. Additionally to the increase of the precision achieved thanks to various GNSS there are many other ways to eliminate so called dead areas like use of IMU systems or pseudolite systems. Many of these redundant ways, allows to get position faster, which spreads the field of their applications. The interesting possibility to augment the positioning technology, especially in areas with permanently limited access to satellite observations, is co working with the systems based on very popular in communication networks RF transmission. In the article a results of preliminary tests with the zigbee radio transceiver for ranging in small closed areas are presented.
\end{abstract}

Keywords: ranging; ZigBee; collaborative navigation; WPAN nets.

\begin{tabular}{|ll}
\hline \multicolumn{2}{|l}{ Nomenclature } \\
WPAN & wireless person area network \\
FFD & full function device \\
RFD & reduced function device \\
DQF & data quality factor \\
TOF & time of fly \\
TOA & time of arrival \\
PMU & Phase diffrence measurement unit \\
\hline
\end{tabular}

\section{Introduction}

Continuous progress in development of electronics and computer sciences gives a new possibilities to improve devices and methods of precise and accurate positioning. Low costs and small dimensions of the modern elements necessary to build positioning systems allow to create them much more complex and effective. Increase in sensitivity more and more modern sensors is very often not enough to design fully functional devices. Only the fast communication and interoperability of many objects can results in useful synergy of the whole systems. Such an idea depicted in Fig. 1 gives measurable effects.

One of the most promising ways to increase accuracy, precision and reliability of positioning systems is information redundancy as a base to reasoning about real position [1]. The redundancy can be considered in the several layers.

The most common approach to get the information required to improve the final result of positioning, is to use the data coming from independent sources based on the schemes of signal acquisition. Classical application of this kind is parallel use of several independent GNSS system such as GPS, GLONAS etc. The significat disadvantage of such approach, is a strong dependency of the sources on the same external conditions just like in case of the loss of all information inside buildings due to shadowing of GNSS satellite signals. A partial solution of such problem is to use signals coming from sensor based on different physical phenomenon and functionality. In this layer, which we could call multisensory layer it is common to use: magnetometers, IMU, WLAN and UWB [3] ranging devices, barometers, image based ranging devices, maps etc. [4]. In more specific applications ultrasound sensors, laser optical sensors etc. are also used. Signals from these sensors thanks to its individual physical properties gain specific advantages in different conditions Classical example of effective coexistence of such different sensors in single device are spreading the field of applications based on the GPS system with IMU sensors. Connection like this allows to continue the positioning in places such as tunnels or shadowed rooms.

Corresponding author: Michał Śmieja. E-mail address: smieja@uwm.edu.pl

http://dx.doi.org/10.3846/enviro.2014.245

(C) 2014 The Author. Published by VGTU Press. This is an open-access article distributed under the terms of the Creative Commons Attribution License, which permits unrestricted use, distribution, and reproduction in any medium, provided the original author and source are credited. 


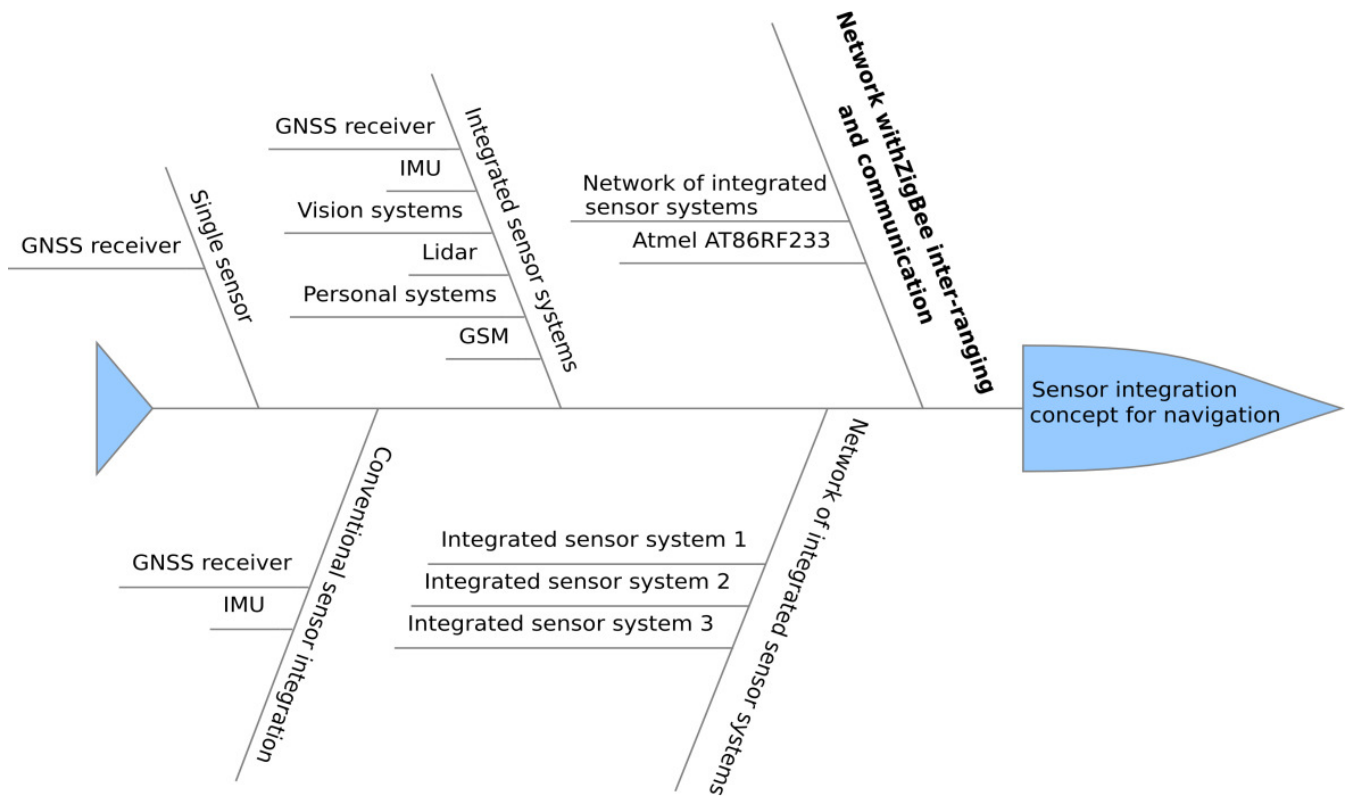

Fig. 1. The development of the positioning systems

Another approach to execute verification of object position, is relative ranging between multiple objects. Such Interranging is proposed e.g. in [5] for GPS pseudolite systems or in [2] for Collaborative Navigation

The integration information coming from various objects makes it possible to get improve in continously accessed data about its current location. Such systems verify the partial sets of information and synthesize the final positioning data

\section{RF ZigBee based ranging}

One of significant alternatives to GNSS in ranging in small areas is based on RF Technology regulated by the IEEE 802.15.4 standard.This standard describes Medium Access Control (MAC) and Physical Layer (PHY) for so called WPAN (Wireless Personal Area Networks). Due to its wideband nature power emission limits are imposed on the transceivers which results in ranging distance limitations. The measuremt technology based on IEEE 802.15.4 uses the received signals as the source of information about: time of arrival (TOA), time difference of arrival (TDOA), Angle of arrival or received signal strenght (RSS).

The interesting ranging solution, applied in devices based on ZigBee protocol is low power, $2.4 \mathrm{GHz}$ transceiver AT86RF233 from ATMEL. Its construction including The Phase Difference Measurement Unit (PMU) makes it possible to perform ranging based on the phase shift measurement as well as time of fly measuremet with TOF module. Its Ultra low current consumption dedicates it to work in nets as a mobile node supplied with a battery for a long time. On board modules serving communication and ranging simplifies hardware and software construction of navigation devices.

Taking into account presented in the introduction tendency to integrate localization sensor systems into sets of objects, the depicted in the Fig. 2 topology of ZigBee seems to be excellent fit to be utilized as a collaborative positioning network.
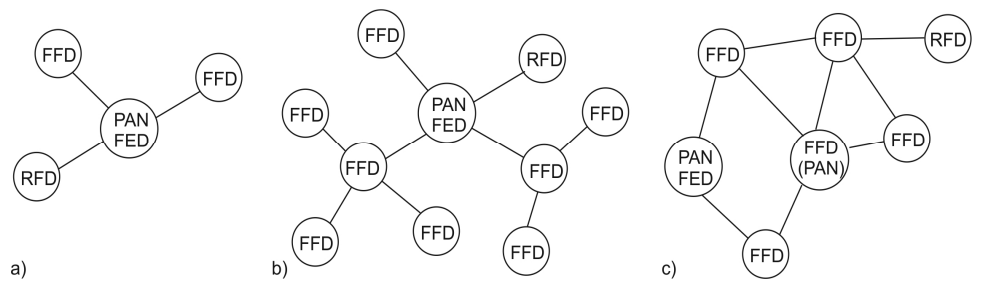

Fig. 2. Topology models supported by ZigBee

Organized in star, cluster tree, and mesh topology ZigBee network consist of components responsible for various activities. The full function device (FFD) operates as a personal area coordinator (PAN), a coordinator or a device. The reduced function device (RFD) has limited functionality to operate as a device. As far as the star and cluster tree topology (Fig. 2a, b) have a secondary meaning for collaborative localization, the mesh topology (Fig. 2c) can be very effectively applied in this matter. The mesh network, called also peer-to-peer network, can be currently reorganized as the most effective configuration. This way every node may communicate and calculate distance to any other node. Many industrial applications confirm reliability and flexibility of this network architecture. 


\section{Tests results}

Preliminary test of ZigBee network in ranging were conducted as a series of measurements with Atmel REB233SMAD Evaluation Kit [6]. REB233SMAD includes the software functionality based on Atmel ranging toolbox (RTB) which architecture is presented in the Fig. 3.

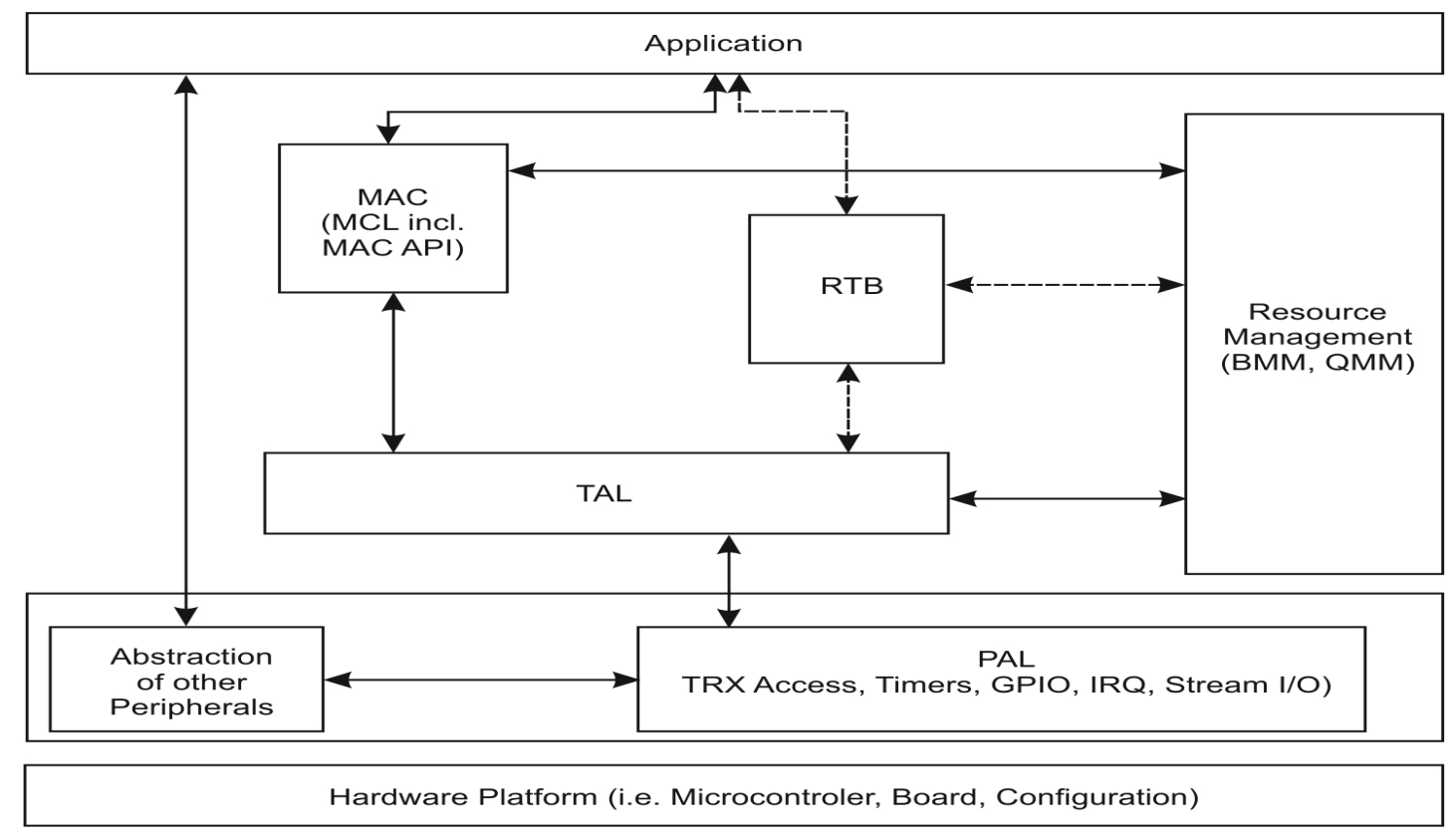

Fig. 3. ZigBeeMac software arhitecture including RTB

The hardware architecture of applied evaluation kit consist of nodes operating as: coordinators, initiators and reflectors. Different available configurations are depicted in Fig. 4 as local ranging (Fig. $4 \mathrm{~b}$ ) and remote ranging (Fig. 4a). The coordinator works as the interface to PC computers and request ranging measurement between initiators and reflectors. The initiator is responsible for signal receive from reflector and for distance calculation.
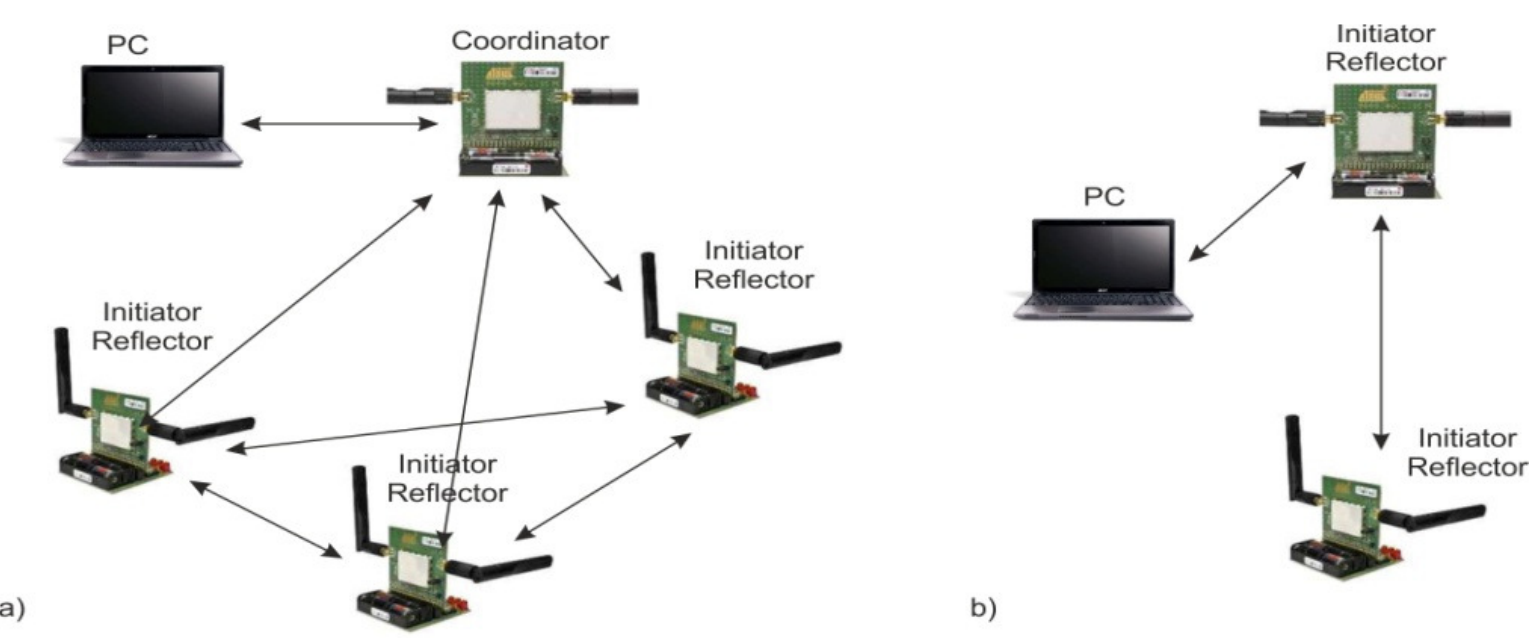

Fig. 4. Remote and local ranging

In the Experiment local ranging was performed. Initiator and reflector were equiped with two antennas what allowed to get four sets of data in every measurement. Each of four sets is associated with a pair of co working antennas as illustrated in Fig. 5.

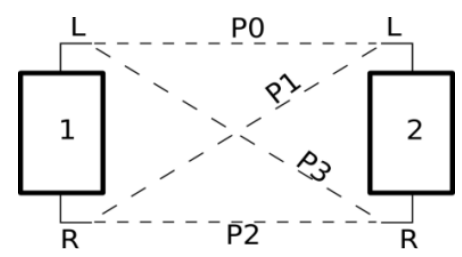

Fig. 5. Configuration of initiator and reflector antennas 
The tests were performed in long, free-of obstacles corridor for distances $2,4,6,8,10$ and $15 \mathrm{~m}$. The average diversities between real distances and measured distances were located in range from 0,2 to $3 \mathrm{~m}$, depending on the measured distance as far as individual pairs are concerned. In many series there were visible relations between drop of DQF and divergence of measurement.

The selected results of the ranging measurements are shown in Fig. 6-9.
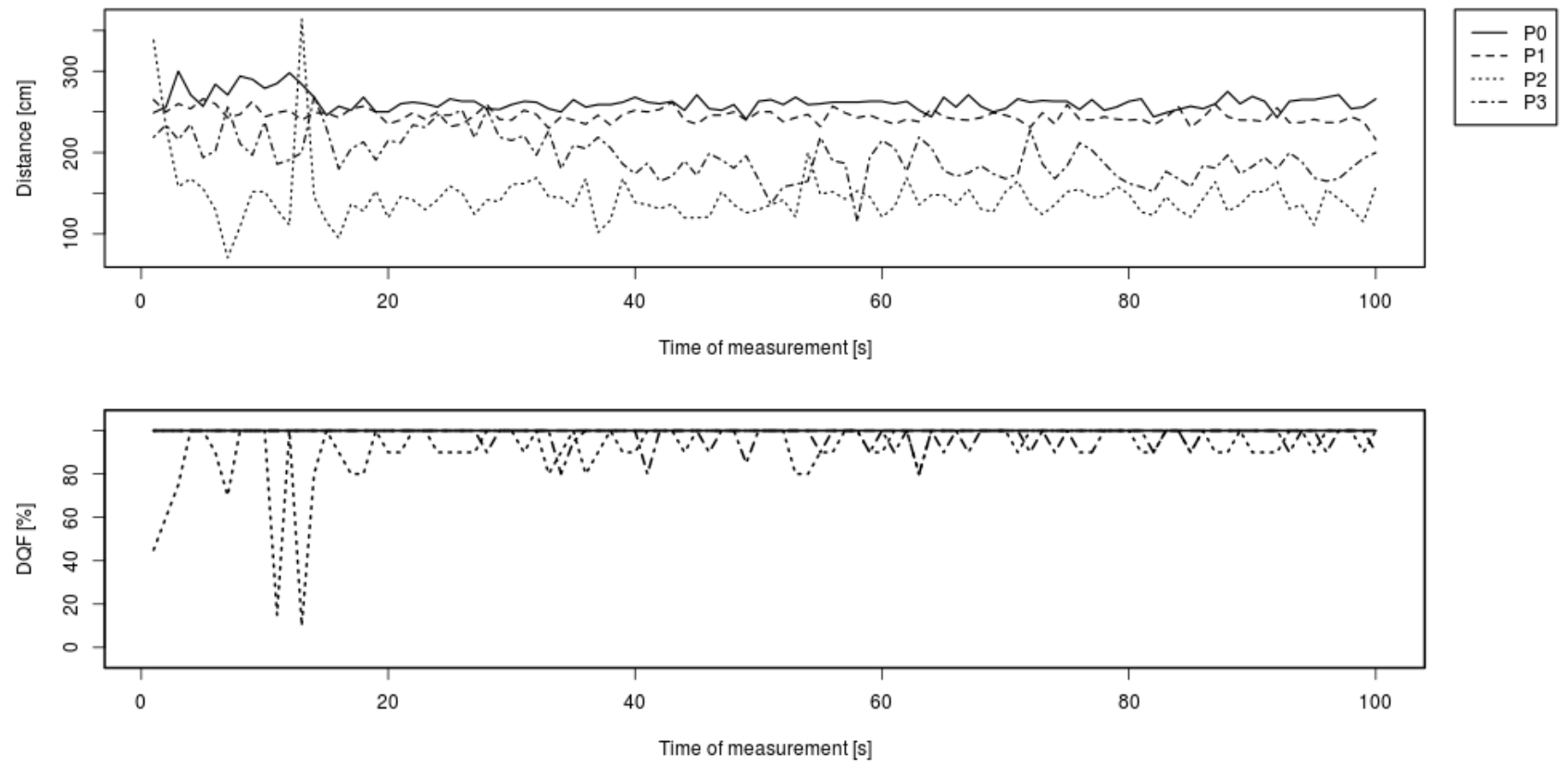

Fig. 6. Ranging measurements and value of data quality factor for distance $2 \mathrm{~m}$
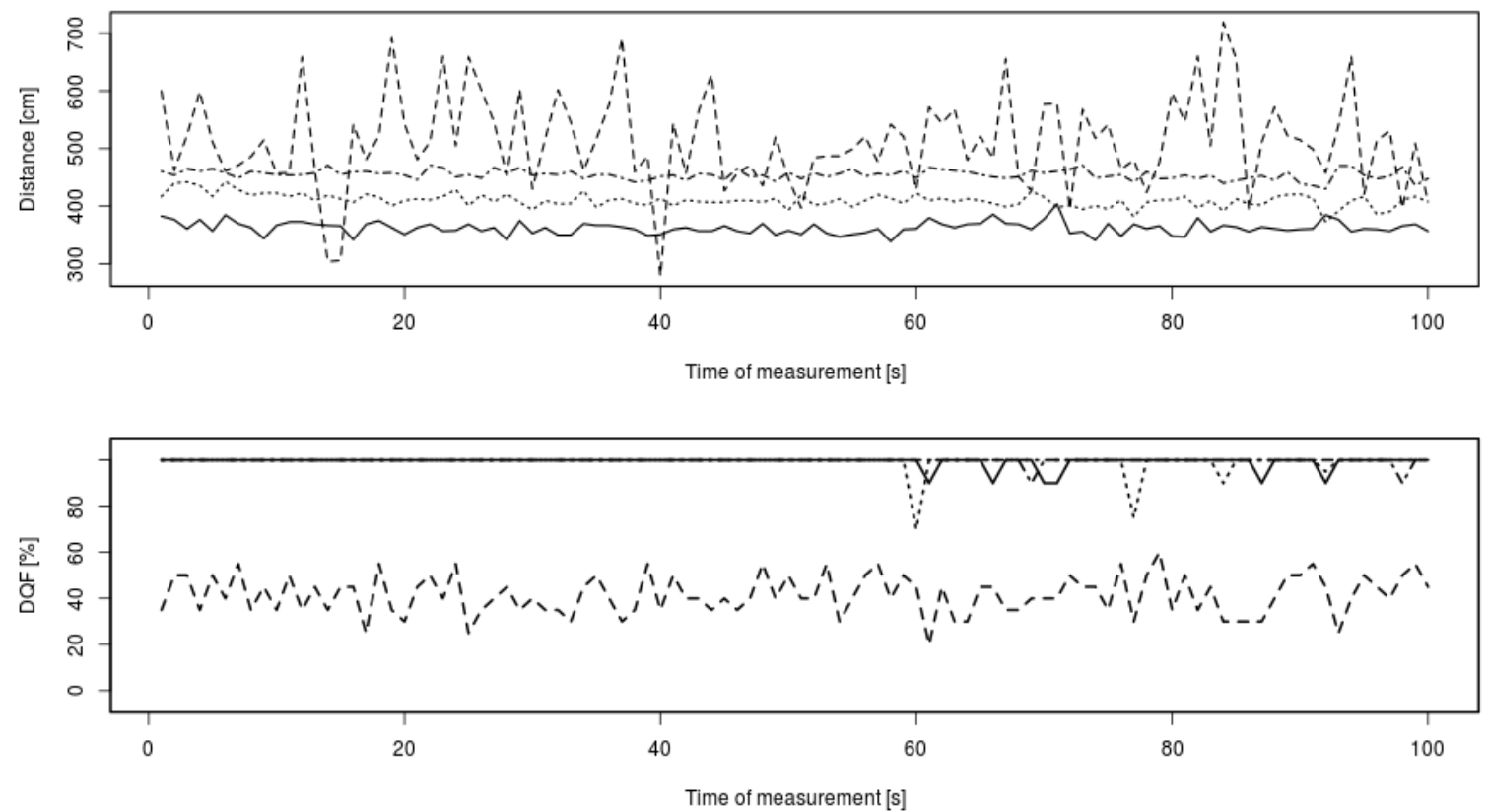

Fig. 7. Ranging measurements and value of data quality factor for distance $4 \mathrm{~m}$ 

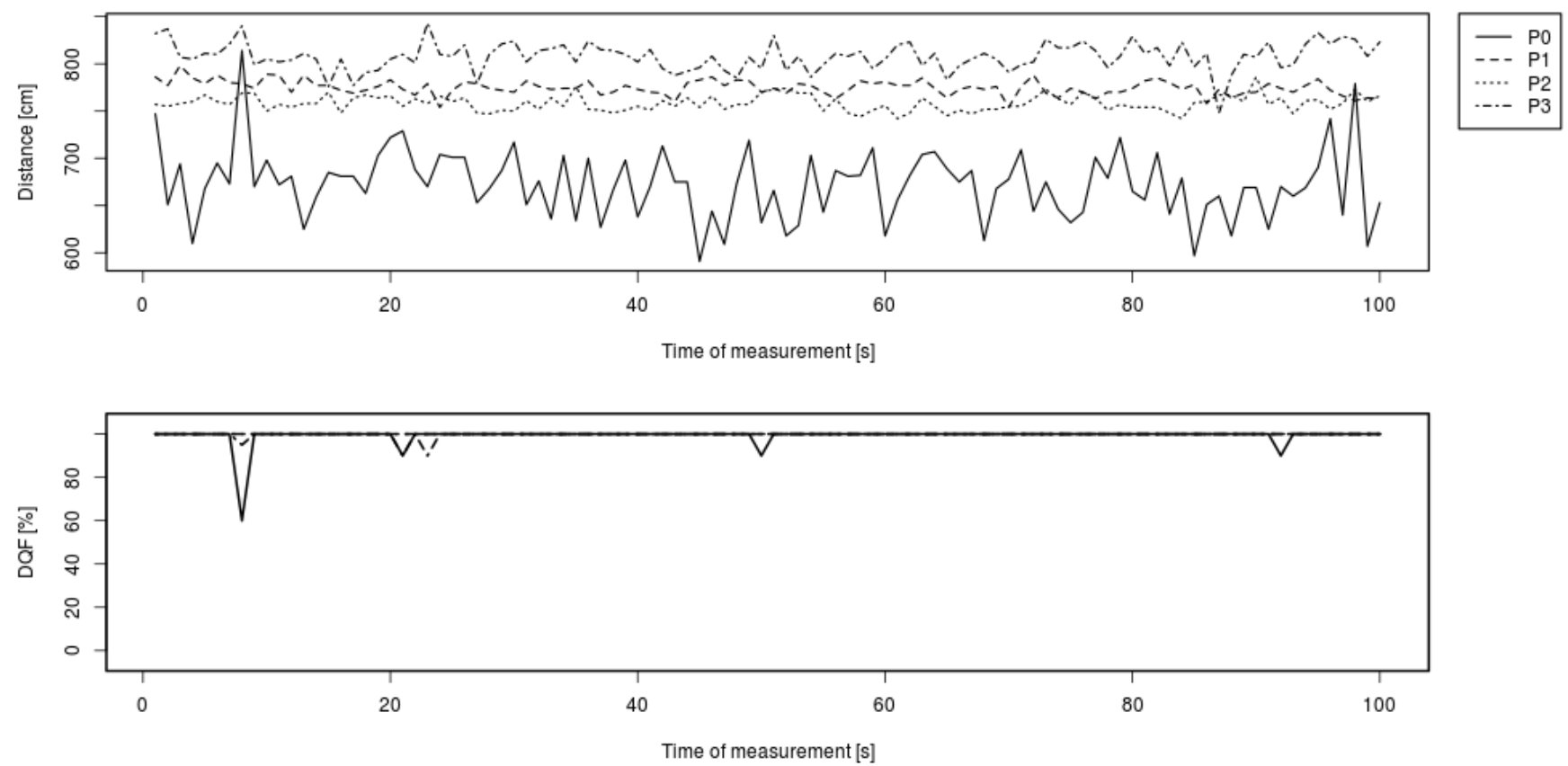

Fig. 8. Ranging measurements and value of data quality factor for distance $8 \mathrm{~m}$
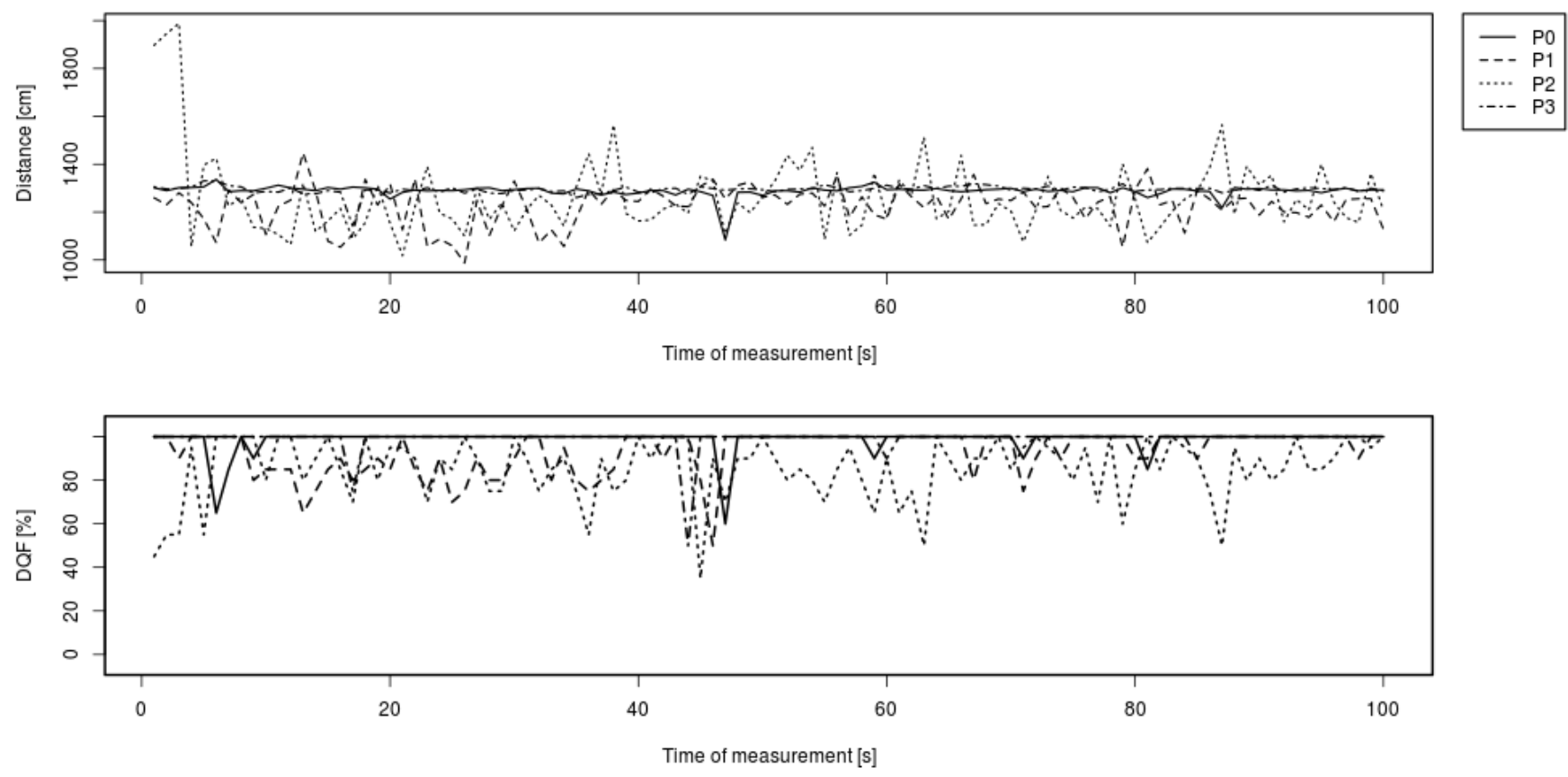

Fig. 9. Ranging measurements and value of data quality factor for distance $12 \mathrm{~m}$

Figures 6 to 9 shows some general tendencies in the results obtained with presented measurement technology. The tendencies are as follows: value of data quality factors (DQF) depends on the distance between initiator and reflector - for longer distances this value drops, what is explicitly visible in Fig. 9 (dist $12 \mathrm{~m}$ ). For shorter distances DQF drops are rather accidental, however its effect on the relative distance is visible in charts for particular antennas pair. In most of cases correlation between chart representing couple of antenna pairs for eg. Pairs pair 1 and pair 2 can be observed. In the Fig. 8 and Fig. 9 the correlation is stronger than in Fig. 7, in this case the strongest correlation is between airs $0-1$ and pair 2-3. The reason of it is multipath of a signal, which is different for different antenna pairs. This way effects of signal multipath varies due to different location of reflector and initiator. The way to avoid it would be to increase the number of nodes, inter-verification and effective distance calculation algorithms. 


\section{Summary}

The preliminary results show the the existence relation between data quality factor and accuracy of measured distance Noisy measurement are signalized by a drop of DQF. The observed divergences between individual pairs are effects of signals multipath. Application of more nodes and preparation of effective algorithms could improve the results of measurement. In further research paralell measurements with PUM and TOF should be performed. Test in room with complex spatial geometry should also be performed. The application of this method of ranging can help in locating moving objects with size of about 1-2 m. Low cost tested device give the possibility to create very numerous network of nodes. Dual functionality of tested transceivers could help with carrying additional information about close environment of the node, what can be especially important for example during the emergency operations.

\section{References}

[1] Grejner-Brzezinska, D. 2013. Current trends in PNT research and applications, in IAG Scientific Assembly Potsdam Germany 2013.

[2] Jong, Ki Lee; Grejner-Brzezinska, D.; Toth, C. 2012. Network -based Collaborative Navigation in GPS-Denied Environment, The Journal of Navigation 65: 445-457. http://dx.doi.org/10.1017/S0373463312000069

[3] Sahinoglu, Z.; Gezici, S.; Guvenc, I. 2008. Ultra-wideband positioning systems. Teoretical limits, ranging algorithms and protocols. Cambridge University Press. http://dx.doi.org/10.1017/CBO9780511541056

[4] Grejner-Brzezinska, D. A.; Toth, Ch.; Moafipoor, S.; Jwa, Y.; Kwo, J. 2006. Multi-sensor personal navigator supported by human motion dynamicd model, in $3^{\text {rd }} I A G / 12^{\text {th }}$ FIG Symposium, Baden, maj 22-24, 2006.

[5] LeMaster, E. A.; Rock, A. M. 2000. Field test results for a self-valibrating pseudolite array. Institute of Navigations, Salt lake City. UT, September, 2000

[6] Atmel AVR 2152. 2013. RTB Evaluation Application Software. User's Guide. Atmel, 2013. 